\title{
Algebraic Local Cohomology Classes Attached to Unimodal Singularities
}

\author{
by
}

Shinichi TAJIma and Yayoi NAKAmURA

\begin{abstract}
Algebraic local cohomology classes and holonomic systems attached to non-quasihomogeneous isolated unimodal singularities are considered in the context of algebraic analysis. Holonomic systems and their algebraic local cohomology solution spaces attached to a unimodal singularity are studied in a constructive manner. The holonomic system constructed from linear partial differential operators of order at most two that annihilate the given algebraic local cohomology is proven to be simple for the case of nonquasihomogeneous unimodal singularities.
\end{abstract}

2010 Mathematics Subject Classification: 14B15, 32C37, 32S25.

Keywords: unimodal singularities, algebraic local cohomology, holonomic D-module.

\section{Introduction}

Let $X$ be a neighborhood of the origin $O$ in $\mathbb{C}^{n}$. Let $\mathcal{H}_{[O]}^{n}\left(\Omega_{X}^{n}\right)$ be the algebraic local cohomology group supported at the origin, where $\Omega_{X}^{n}$ is the sheaf on $X$ of holomorphic differential $n$-forms. Let $f$ be a holomorphic function on $X$ with an isolated singularity at the origin. Denote by $\mathcal{W}_{f}$ the set of algebraic local cohomology classes in $\mathcal{H}_{[O]}^{n}\left(\Omega_{X}^{n}\right)$ that are annihilated by the Jacobi ideal $\mathcal{I} \subset \mathcal{O}_{X, O}$ of $f$, and let $\omega$ be a generator of $\mathcal{W}_{f}$ over $\mathcal{O}_{X, O}$, where $\mathcal{O}_{X, O}$ is the stalk at the origin of the sheaf $\mathcal{O}_{X}$ of holomorphic functions on $X$.

Inspired by a result obtained by K. Saito [7], we previously studied algebraic local cohomology classes attached to quasihomogeneous hypersurface isolated singularities in the category of left $\mathcal{D}_{X}$-modules [8]. We showed in particular that

Communicated by M. Kashiwara. Received May 31, 2010. Revised September 22, 2010.

S. Tajima: Graduate School of Pure and Applied Sciences, University of Tsukuba, Tenodai 1-1-1, Tsukuba, Ibaraki 305-8571, Japan;

e-mail: tajima@math.tsukuba.ac.jp

Y. Nakamura: School of Science and Engineering, Kinki University, 3-4-1, Kowakae, Higashiosaka, Osaka 577-8502, Japan;

e-mail: yayoi@math.kindai.ac.jp

(C) 2012 Research Institute for Mathematical Sciences, Kyoto University. All rights reserved. 
the quasihomogeneity of hypersurface isolated singularities can be characterized in terms of the holonomic $\mathcal{D}_{X}$-modules. To be more precise, let $\mathcal{A} n n_{\mathcal{D}_{X, O}}(\omega)$ be the annihilator ideal of $\omega \in \mathcal{W}_{f}$ in the stalk $\mathcal{D}_{X, O}$ at the origin of the sheaf $\mathcal{D}_{X}$ of rings of linear partial differential operators. Let $\mathcal{A} n n_{\mathcal{D}_{X, O}}^{(1)}(\omega)$ denote the right ideal in $\mathcal{D}_{X, O}$ generated by differential operators of order at most 1 that annihilate $\omega$. Then the result can be stated in terms of right $\mathcal{D}_{X, O}$-modules as follows.

Theorem 0.1 (cf. [8]). Let $X$ be an open neighborhood of the origin in $\mathbb{C}^{n}$. Let

$$
f=f\left(x_{1}, \ldots, x_{n}\right) \in \mathcal{O}_{X, O}
$$

be a holomorphic function with an isolated singularity at the origin and denote by $\omega$ a generator of $\mathcal{W}_{f}$ over $\mathcal{O}_{X, O}$. The following conditions are equivalent:

(i) $\mathcal{O}_{X, O}\left(f, \frac{\partial f}{\partial x_{1}}, \ldots, \frac{\partial f}{\partial x_{n}}\right)=\mathcal{O}_{X, O}\left(\frac{\partial f}{\partial x_{1}}, \ldots, \frac{\partial f}{\partial x_{n}}\right)$.

(ii) $\mathcal{A} n n_{\mathcal{D}_{X, O}}(\omega)$ is generated by differential operators of order at most 1 , i.e., $\mathcal{A} n n_{\mathcal{D}_{X, O}}(\omega)=\mathcal{A} n n_{\mathcal{D}_{X, O}}^{(1)}(\omega)$.

(iii) The holonomic $\mathcal{D}_{X, O}$-module $\mathcal{D}_{X, O} / \mathcal{A} n n_{\mathcal{D}_{X, O}}^{(1)}(\omega)$ is simple.

Indeed, the above theorem can be proved, as done previously in [8], by showing in the category of right $\mathcal{D}_{X}$-modules that if a given hypersurface isolated singularity is non-quasihomogeneous, then the dimension of the algebraic local cohomology solution space

$$
\mathcal{H} m_{\mathcal{O}_{X}}\left(\mathcal{D}_{X, O} / \mathcal{A} n n_{\mathcal{D}_{X, O}}^{(1)}(\omega), \mathcal{H}_{[O]}^{n}\left(\Omega_{X}^{n}\right)\right)
$$

to the holonomic system $\mathcal{D}_{X, O} / \mathcal{A} n n_{\mathcal{D}_{X, O}}^{(1)}(\omega)$ is greater than or equal to 2 . However, the structure of the holonomic system $\mathcal{D}_{X, O} / \mathcal{A} n n_{\mathcal{D}_{X, O}}^{(1)}(\omega)$ and its algebraic local cohomology solution space associated with the non-quasihomogeneous isolated singularity have not yet been determined, even for the most typical case.

In this paper, we study the case of non-quasihomogeneous hypersurface isolated singularities. We consider the right ideal $\mathcal{A} n n_{\mathcal{D}_{X, O}}^{(k)}(\omega), k=0,1,2, \ldots$, generated by differential operators of order at most $k$ and holonomic systems $\mathcal{D}_{X, O} / \mathcal{A} n n_{\mathcal{D}_{X, O}}^{(k)}(\omega)$ attached to non-quasihomogeneous hypersurface isolated singularities. We investigate in particular unimodal singularities, the most typical case among hypersurface singularities, that comprise hyperbolic singularities and 14 exceptional families [1]. By extending the algorithms introduced in earlier work $[9],[10]$ to the parametric case for computing algebraic local cohomology and annihilators, we explicitly determine the algebraic local cohomology solution space

$$
\mathcal{H}_{o m} \overline{\mathcal{D}}_{X, O}\left(\mathcal{D}_{X, O} / \mathcal{A n n}_{\mathcal{D}_{X, O}}^{(k)}(\omega), \mathcal{H}_{[O]}^{n}\left(\Omega_{X}^{n}\right)\right)
$$


for the non-quasihomogeneous unimodal singularity case. We show as an application that the multiplicity of the holonomic system $\mathcal{D}_{X, O} / \mathcal{A} n n_{\mathcal{D}_{X, O}}^{(1)}(\omega)$ is equal to 2 and that of $\mathcal{D}_{X, O} / \mathcal{A} n n_{\mathcal{D}_{X, O}}^{(2)}(\omega)$ is equal to 1 .

In Section 1, after introducing some basic notions, we associate a series of holonomic systems $\mathcal{D}_{X, O} / \mathcal{A} n n_{\mathcal{D}_{X, O}}^{(k)}(\omega), k=0,1, \ldots$, to hypersurface isolated singularities. We show that the multiplicities of the holonomic systems are analytical invariants of the hypersurface isolated singularities. In Section 2, we study basic properties of algebraic local cohomology solution spaces for $\mathcal{A} n n_{\mathcal{D}_{X, O}}^{(k)}(\omega)$ and state the main results. In Section 3, we consider hyperbolic singularities and prove the main result by explicitly providing $\mathcal{W}_{f}$ and annihilators. In Section 4 , we study exceptional singularities by adopting a constructive approach. Instead of presenting the full set of data on $\mathcal{W}_{f}$, a generator $\omega$ and annihilators for each case, we examine the $Z_{13}$ singularity and give the results of computations to illustrate the proof.

\section{$\S 1$. Holonomic systems attached to hypersurface isolated singularities}

Let $X$ be an open neighborhood of the origin $O$ in $\mathbb{C}^{n}$. Denote by $\mathcal{D}_{X, O}$ the stalk at $O$ of the sheaf $\mathcal{D}_{X}$ of holomorphic linear partial differential operators on $X$. Let $\mathcal{H}_{[O]}^{n}\left(\Omega_{X}^{n}\right)$ be the algebraic local cohomology group supported at $O$, where $\Omega_{X}^{n}$ is the sheaf of holomorphic differential $n$-forms on $X$. Note that, since $\Omega_{X}^{n}$ has the structure of the right $\mathcal{D}_{X, O}$-module, differential operators in $\mathcal{D}_{X, O}$ act on algebraic local cohomology classes in $\mathcal{H}_{[O]}^{n}\left(\Omega_{X}^{n}\right)$ from the right.

Definition 1.1. For an algebraic local cohomology class $\zeta \in \mathcal{H}_{[O]}^{n}\left(\Omega_{X}^{n}\right)$, let $\mathcal{L}_{\mathcal{D}_{X, O}}^{(k)}(\zeta)$ be the set of germs of linear partial differential operators of order at most $k$ that annihilate $\zeta$. We define $\mathcal{A} n n_{\mathcal{D}_{X, O}}^{(k)}(\zeta)$ to be the right ideal in $\mathcal{D}_{X, O}$ generated by $\mathcal{L}_{\mathcal{D}_{X, O}}^{(k)}(\zeta)$ :

$$
\mathcal{L}_{\mathcal{D}_{X, O}}^{(k)}(\zeta)=\left\{P \in \mathcal{D}_{X, O} \mid \zeta P=0, \text { ord } P \leq k\right\}, \quad \mathcal{A} n n_{\mathcal{D}_{X, O}}^{(k)}(\zeta)=\mathcal{L}_{\mathcal{D}_{X, O}}^{(k)}(\zeta) \mathcal{D}_{X, O} .
$$

Denote by $\mathcal{O}_{X, O}$ the stalk at $O$ of the sheaf $\mathcal{O}_{X}$ of holomorphic functions on $X$. Let $f=f\left(x_{1}, \ldots, x_{n}\right)$ be a holomorphic function on $X$, defining an isolated singularity at the origin, and $\mathcal{I}$ the Jacobi ideal of $f$ in $\mathcal{O}_{X, O}$ :

$$
\mathcal{I}=\mathcal{O}_{X, O}\left(\frac{\partial f}{\partial x_{1}}, \ldots, \frac{\partial f}{\partial x_{n}}\right)
$$

Let $\mathcal{W}_{f}$ be the set of algebraic local cohomology classes annihilated by the Jacobi ideal $\mathcal{I}$ :

$$
\mathcal{W}_{f}=\left\{\eta \in \mathcal{H}_{[O]}^{n}\left(\Omega_{X}^{n}\right) \mid g \eta=0 \text { for all } g \in \mathcal{I}\right\}
$$


Then $\mathcal{W}_{f}$ is a $\mu$-dimensional vector space, where $\mu$ is the Milnor number $\operatorname{dim}_{\mathbb{C}} \mathcal{O}_{X, O} / \mathcal{I}$

$\mathcal{W}_{f}$ is generated, over $\mathcal{O}_{X, O}$, by one algebraic local cohomology class. Though the choice of a generator of $\mathcal{W}_{f}$ over $\mathcal{O}_{X, O}$ is not unique, we have the following.

Lemma 1.1. Let $\omega$ and $\omega^{\prime}$ be two generators of $\mathcal{W}_{f}$ over $\mathcal{O}_{X, O}$, that is,

$$
\mathcal{W}_{f}=\mathcal{O}_{X, O} \omega=\mathcal{O}_{X, O} \omega^{\prime}
$$

Then the $\mathcal{D}_{X, O}$-modules $\mathcal{D}_{X, O} / \mathcal{A} n n_{\mathcal{D}_{X, O}}^{(k)}(\omega)$ and $\mathcal{D}_{X, O} / \mathcal{A} n n_{\mathcal{D}_{X, O}}^{(k)}\left(\omega^{\prime}\right)$ are isomorphic.

Proof. There is a holomorphic function $g=g(x) \in \mathcal{O}_{X, O}$ with $g(0) \neq 0$ such that $\omega=g \omega^{\prime}$. Let $\phi: \mathcal{D}_{X, O} \rightarrow \mathcal{D}_{X, O}$ be a $\mathcal{D}_{X, O}$-linear homomorphism defined by $\phi(R)=g R$ for $R \in \mathcal{D}_{X, O}$. Then $\phi$ induces an isomorphism from $\mathcal{D}_{X, O} / \mathcal{A} n n_{\mathcal{D}_{X, O}}^{(k)}(\omega)$ to $\mathcal{D}_{X, O} / \mathcal{A} n n_{\mathcal{D}_{X, O}}^{(k)}\left(\omega^{\prime}\right)$.

It is known that the right $\mathcal{D}_{X, O}$-module $\mathcal{D}_{X, O} / \mathcal{A} n n_{\mathcal{D}_{X, O}}^{(k)}(\zeta)$ is a holonomic system with support at the origin, for any $\zeta \in \mathcal{H}_{[O]}^{n}\left(\Omega_{X}^{n}\right)$. The next statement immediately follows from Lemma 1.1.

Lemma 1.2. Let $\omega$ and $\omega^{\prime}$ be two generators of $\mathcal{W}_{f}$ over $\mathcal{O}_{X, O}$. Then the multiplicity of the holonomic system $\mathcal{D}_{X, O} / \mathcal{A n n}_{\mathcal{D}_{X, O}}^{(k)}(\omega)$ is equal to the multiplicity of $\mathcal{D}_{X, O} / \mathcal{A} n n_{\mathcal{D}_{X, O}}^{(k)}\left(\omega^{\prime}\right)$.

That is, the multiplicity of the holonomic system $\mathcal{D}_{X, O} / \mathcal{A} n n_{\mathcal{D}_{X, O}}^{(k)}(\omega)$ is independent of the choice of a generator of $\mathcal{W}_{f}$. We thus arrive at the next definition.

Definition 1.2. We denote by $\mu_{f}^{(k)}$ the multiplicity of the holonomic system $\mathcal{D}_{X, O} / \mathcal{A} n n_{\mathcal{D}_{X, O}}^{(k)}(\omega)$, where $\omega$ is a generator of $\mathcal{W}_{f}$ over $\mathcal{O}_{X, O}$.

Note that if we denote the conormal space of the origin $O \in X$ by $T_{\{O\}}^{*} X$, then the characteristic cycle of the holonomic system $\mathcal{D}_{X, O} / \mathcal{A} n n_{\mathcal{D}_{X, O}}^{(k)}(\omega)$ is given by $\mu_{f}^{(k)} T_{\{O\}}^{*} X$.

Let $\tilde{f}(x, t)$ be a function on $\tilde{X}=X \times T$ of the form $\tilde{f}(x, t)=f(x)+$ $t_{1}^{2}+\cdots+t_{\ell}^{2}$ with $t=\left(t_{1}, \ldots, t_{\ell}\right) \in T$, where $T$ is an open neighborhood of the origin in $\mathbb{C}^{\ell}$. Let $\mathcal{W}_{\tilde{f}} \subset \mathcal{H}_{[O]}^{n+\ell}\left(\Omega_{\tilde{X}}^{n+\ell}\right)$ be the set of algebraic local cohomology classes with support at the origin $O$ of $\tilde{X}$ annihilated by the Jacobi ideal $\mathcal{O}_{\tilde{X}, O}\left(\partial f / \partial x_{1}, \ldots, \partial f / \partial x_{n}, t_{1}, \ldots, t_{\ell}\right)$ of $\tilde{f}(x, t)$. 
Lemma 1.3. Let $\tilde{\omega}$ be a generator of $\mathcal{W}_{\tilde{f}}$ over $\mathcal{O}_{\tilde{X}, O}$. Then there exists an algebraic local cohomology class $\omega \in \mathcal{W}_{f}$ such that $\tilde{\omega}=\omega \otimes \delta_{T}$, where $\delta_{T}$ is the delta function with support at the origin of T. Furthermore,

$$
\mathcal{A} n n_{\mathcal{D}_{\tilde{X}, O}}^{(k)}(\tilde{\omega})=\mathcal{A} n n_{\mathcal{D}_{X, O}}^{(k)}(\omega) \mathcal{D}_{\tilde{X}, O}+\left(t_{1}, \ldots, t_{\ell}\right) \mathcal{D}_{\tilde{X}, O} .
$$

Theorem 1.1. Let $\tilde{f}=\tilde{f}(x, t)$ be a holomorphic function defined on $\tilde{X}=X \times T$. Assume that $\tilde{f}$ is stably equivalent to $f$. Then $\mu_{\tilde{f}}^{(k)}=\mu_{f}^{(k)}$ for every $k \in \mathbb{N}$, and $\nu_{\tilde{f}}=\nu_{f}$.

Proof. If two holomorphic functions defined on $\tilde{X}$ are right equivalent, then the multiplicities of the corresponding holonomic systems defined by $k$ th-order annihilators are equal. Therefore, to prove the theorem, it suffices to consider $\tilde{f}$ of the form $\tilde{f}(x, t)=f(x)+t_{1}^{2}+\cdots+t_{\ell}^{2}$. Let $\tilde{\omega}$ be a generator of $\mathcal{W}_{\tilde{f}}$ over $\mathcal{O}_{\tilde{X}, O}$. Then $\tilde{\omega}$ can be written in the form $\tilde{\omega}=\omega \otimes \delta_{T}$ with some generator $\omega \in \mathcal{H}_{[O]}^{n}\left(\Omega_{X}^{n}\right)$ of $\mathcal{W}_{f}$. Lemma 1.3 also implies that

$$
\mathcal{D}_{\tilde{X}, O} / \mathcal{A} n n_{\mathcal{D}_{X, O}}^{(k)}(\tilde{\omega}) \cong \mathcal{D}_{X, O} / \mathcal{A} n n_{\mathcal{D}_{X, O}}^{(k)}(\omega) \otimes_{\mathcal{D}_{\tilde{X}, O}} \mathcal{D}_{T, O} /\left(t_{1}, \ldots, t_{\ell}\right) \mathcal{D}_{T, O},
$$

which yields the equality $\mu_{\tilde{f}}^{(k)}=\mu_{f}^{(k)}$.

By Theorem 1.1, $\mu_{f}^{(k)}, k=1,2, \ldots$, are analytical invariants of a given singularity defined by the function $f$.

Since $\left\{\mathcal{A} n n_{\mathcal{D}_{X, O}}^{(k)}(\omega)\right\}_{k}$ is an increasing sequence of right ideals, $\left\{\mu_{f}^{(k)}\right\}_{k}$ becomes a decreasing sequence of positive integers:

$$
\mu_{f}^{(0)} \geq \mu_{f}^{(1)} \geq \cdots \geq \mu_{f}^{(k)} \geq \cdots \geq 1 .
$$

There exists an integer $m$ such that $\mathcal{A} n n_{\mathcal{D}_{X, O}}^{(m)}(\omega)$ is equal to the right ideal $\mathcal{A} n n_{\mathcal{D}_{X, O}}(\omega)$ of $\mathcal{D}_{X, O}$ consisting of linear partial differential operators that annihilate $\omega$. Since the holonomic system $\mathcal{D}_{X, O} / \mathcal{A} n n_{\mathcal{D}_{X, O}}(\omega)$ is simple, we have $\mu_{f}^{(m)}=1$.

Example. Since $\mathcal{A} n n_{\mathcal{D}_{X, O}}^{(0)}(\omega)=\mathcal{I} \mathcal{D}_{X, O}, \mu_{f}^{(0)}$ is the Milnor number $\operatorname{dim}_{\mathbb{C}} \mathcal{O}_{X, O} / \mathcal{I}$ at the origin of the isolated hypersurface singularity $f$.

Example. As seen in Theorem $0.1, \mu_{f}^{(k)}=1$ for $k \geq 1$ in quasihomogeneous cases and $\mu_{f}^{(1)} \geq 2$ in non-quasihomogeneous cases.

Let $B$ be the $\mu$-dimensional vector space spanned by a monomial basis of the quotient space $\mathcal{O}_{X, O} / \mathcal{I}$. If we set

$$
L_{\mathcal{D}_{X}}^{(1)}(\omega)=\left\{P=\sum_{i=1}^{n} a_{i}(x) \frac{\partial}{\partial x_{i}}+a_{0}(x) \mid \omega P=0, a_{i}(x) \in B, i=0,1, \ldots, n\right\},
$$


then

$$
\mathcal{A} n n_{\mathcal{D}_{X, O}}^{(1)}(\omega)=L_{\mathcal{D}_{X}}^{(1)}(\omega) \mathcal{D}_{X, O}+\mathcal{I D}_{X, O}
$$

If we set

$$
\begin{aligned}
L_{\mathcal{D}_{X}}^{(2)}(\omega)=\left\{P=\sum_{i, j=1}^{n} a_{i, j}(x) \frac{\partial^{2}}{\partial x_{i} \partial x_{j}}+\sum a_{i}(x) \frac{\partial}{\partial x_{i}}+a_{0}(x) \mid\right. \\
\left.\omega P=0, a_{i j}(x) \in B, a_{i}(x) \in B, i, j=1, \ldots, n, a_{0}(x) \in B\right\}
\end{aligned}
$$

then also

$$
\mathcal{A} n n_{\mathcal{D}_{X, O}}^{(2)}(\omega)=L_{\mathcal{D}_{X}}^{(2)}(\omega) \mathcal{D}_{X, O}+L_{\mathcal{D}_{X}}^{(1)}(\omega) \mathcal{D}_{X, O}+\mathcal{I D}_{X, O}
$$

(see [4]). Accordingly, generators of $\mathcal{A} n n_{\mathcal{D}_{X, O}}^{(1)}(\omega)$ and of $\mathcal{A} n n_{\mathcal{D}_{X, O}}^{(2)}(\omega)$ can be constructed by utilizing bases of the finite-dimensional vector spaces $L_{\mathcal{D}_{X}}^{(1)}(\omega)$ and $L_{\mathcal{D}_{X}}^{(2)}(\omega)$. Based on this observation, we have recently derived an efficient algorithm for computing the right ideal $\mathcal{A} n n_{\mathcal{D}_{X, O}}^{(k)}(\omega)$ generated by annihilating differential operators of order at most $k$ (see [9]). We also implemented the resulting algorithm in the computer algebra system Risa/Asir [6].

\section{§2. Main result}

Let $\operatorname{Sol}_{f, \omega}^{(k)}$ denote the algebraic local cohomology solution space of the system of partial differential equations defined by the ideal $\mathcal{A} n n_{\mathcal{D}_{X, O}}^{(k)}(\omega)$ :

$$
S o l_{f, \omega}^{(k)}=\left\{\zeta \in \mathcal{H}_{[O]}^{n}\left(\Omega_{X}^{n}\right) \mid \zeta P=0, \forall P \in \mathcal{A} n n_{\mathcal{D}_{X, O}}^{(k)}(\omega)\right\} .
$$

Proposition 2.1. Let $f$ be a holomorphic function with a hypersurface isolated singularity at the origin. Let $\omega$ be a generator of $\mathcal{W}_{f}$. Then

$$
\mu_{f}^{(k)}=\operatorname{dim}_{\mathbb{C}} S o l_{f, \omega}^{(k)} .
$$

Proof. Since the holonomic $\mathcal{D}_{X, O}$-module $\mathcal{D}_{X, O} / \mathcal{A} n n_{\mathcal{D}_{X, O}}^{(k)}(\omega)$ is supported at the origin, we have $\mathcal{E}_{x} t_{\mathcal{D}_{X, O}}^{j}\left(\mathcal{D}_{X, O} / \mathcal{A} n n_{\mathcal{D}_{X, O}}^{(k)}(\omega), \mathcal{H}_{[O]}^{n}\left(\Omega_{X}^{n}\right)\right)=0$ for all $j \geq 1$, which implies

$$
\mu_{f}^{(k)}=\operatorname{dim}_{\mathbb{C}} \mathcal{H} \operatorname{mom}_{\mathcal{D}_{X, O}}\left(\mathcal{D}_{X, O} / \mathcal{A} n n_{\mathcal{D}_{X, O}}^{(k)}(\omega), \mathcal{H}_{[O]}^{n}\left(\Omega_{X}^{n}\right)\right)
$$

The isomorphism

$$
\mathcal{H o m}_{\mathcal{D}_{X, O}}\left(\mathcal{D}_{X, O} / \mathcal{A n n}_{\mathcal{D}_{X, O}}^{(k)}(\omega), \mathcal{H}_{[O]}^{n}\left(\Omega_{X}^{n}\right)\right) \cong \operatorname{Sol}_{f, \omega}^{(k)}
$$

yields the result. 
Lemma 2.1. Let $f$ be a holomorphic function with an isolated singularity at the origin. Let $\omega$ be a generator of $\mathcal{W}_{f}$. Then

(i) $\operatorname{Sol}_{f, \omega}^{(0)}=\mathcal{W}_{f}$.

(ii) $\operatorname{Sol}_{f, \omega}^{(k)} \subset \operatorname{Sol}_{f, \omega}^{(k-1)}$ for any $k \geq 1$.

Proof. By the definition of $\mathcal{A} n n_{\mathcal{D}_{X, O}}^{(k)}(\omega)$, we have

$$
\operatorname{Sol}_{f, \omega}^{(k)}=\left\{\zeta \in \mathcal{H}_{[O]}^{n}\left(\Omega_{X}^{n}\right) \mid \zeta P=0, \forall P \in \mathcal{L}_{\mathcal{D}_{X, O}}^{(k)}(\omega)\right\} .
$$

(i) Since $\mathcal{L}_{\mathcal{D}_{X, O}}^{(0)}(\omega)=\mathcal{I}=\mathcal{O}_{X, O}\left(\partial f / \partial x_{1}, \ldots, \partial f / \partial x_{n}\right)$, we have

$$
S o l_{f, \omega}^{(0)}=\left\{\zeta \in \mathcal{H}_{[O]}^{n}\left(\Omega_{X}^{n}\right) \mid \frac{\partial f}{\partial x_{1}} \zeta=\cdots=\frac{\partial f}{\partial x_{n}} \zeta=0\right\},
$$

which is equal to $\mathcal{W}_{f}$.

(ii) From $\mathcal{A} n n_{\mathcal{D}_{X, O}}^{(k-1)}(\omega) \subset \mathcal{A} n n_{\mathcal{D}_{X, O}}^{(k)}(\omega)$, we have $\operatorname{Sol}_{f, \omega}^{(k)} \subset S o l_{f, \omega}^{(k-1)}$.

The lemma above immediately yields the following description:

$$
\operatorname{Sol}_{f, \omega}^{(k)}=\left\{\zeta \in \operatorname{Sol}_{f, \omega}^{(k-1)} \mid \zeta P=0, \forall P \in \mathcal{L}_{\mathcal{D}_{X, O}}^{(k)}(\omega)\right\}
$$

and

$$
S o l_{f, \omega}^{(k)}=\left\{\zeta \in \mathcal{W}_{f} \mid \zeta P=0, \forall P \in \mathcal{L}_{\mathcal{D}_{X, O}}^{(k)}(\omega)\right\} .
$$

Since $\omega \in \operatorname{Sol}_{f, \omega}^{(k)}$ for any $k$, we have the following.

Proposition 2.2. The following conditions are equivalent:

(i) $\mu_{f}^{(k)}=1$.

(ii) $\mathcal{A} n n_{\mathcal{D}_{X, O}}^{(k)}(\omega)=\mathcal{A} n n_{\mathcal{D}_{X, O}}(\omega)$.

(iii) $\operatorname{Sol}_{f, \omega}^{(k)}=\operatorname{Span}_{\mathbb{C}}\{\omega\}$.

Here, we study holonomic systems and their algebraic local cohomology solutions attached to non-quasihomogeneous unimodal isolated singularities: hyperbolic singularities and exceptional families. Our main results are the following theorems.

Theorem 2.1. Let $f$ be a holomorphic function with a non-quasihomogeneous unimodal isolated singularity at the origin $O$.

(i) $\operatorname{Sol}_{f, \omega}^{(1)}=\operatorname{Span}_{\mathbb{C}}\{\omega, \delta\}$, where $\delta$ is the delta function in $\mathcal{H}_{[O]}^{n}\left(\Omega_{X}^{n}\right)$.

(ii) $\operatorname{Sol}_{f, \omega}^{(2)}=\operatorname{Span}_{\mathbb{C}}\{\omega\}$. 
Remark. It has been shown that $\operatorname{Sol}_{f, \omega}^{(1)} \supseteq \operatorname{Span}_{\mathbb{C}}\{\omega, f \omega\}$ for any non-quasihomogeneous hypersurface isolated singularity [8]. Since the ideal quotient $\mathcal{I}: f$ for the non-quasihomogeneous unimodal function $f$ is the maximal ideal in $\mathcal{O}_{X, O}, f \omega$ coincides with a constant multiple of the delta function $\delta$. Therefore, the inclusion $\operatorname{Sol}_{f, \omega}^{(1)} \supseteq \operatorname{Span}_{\mathbb{C}}\{\omega, \delta\}$ was implicitly given in [8].

Theorem 2.2. Let $f$ be a holomorphic function with a non-quasihomogeneous unimodal isolated singularity at the origin $O$.

(i) The multiplicity of the holonomic system $\mathcal{D}_{X, O} / \mathcal{A} n n_{\mathcal{D}_{X, O}}^{(1)}(\omega)$ is equal to 2.

(ii) The multiplicity of the holonomic system $\mathcal{D}_{X, O} / \mathcal{A n n}_{\mathcal{D}_{X, O}}^{(2)}(\omega)$ is equal to 1.

Theorem 2.2 is a consequence of Theorem 2.1 in combination with Proposition 2.1. By Proposition 2.2, the second statement of Theorem 2.2 can be rephrased as follows.

Corollary 2.1. Let $f \in \mathcal{O}_{X, O}$ be a holomorphic function that defines a nonquasihomogeneous unimodal isolated singularity at the origin. Then

$$
\mathcal{A} n n_{\mathcal{D}_{X, O}}^{(2)}(\omega)=\mathcal{A} n n_{\mathcal{D}_{X, O}}(\omega) .
$$

In other words, the annihilator ideal $\mathcal{A} n n_{\mathcal{D}_{X, O}}(\omega)$ can be generated by annihilating operators of order at most 2 for any generator $\omega$ of the dual space $\mathcal{W}_{f}$ of the Milnor algebra of unimodal singularities.

\section{§3. Hyperbolic singularities}

The normal form of the hyperbolic singularity $T_{p q r}$ is given by the polynomial

$$
f=x^{p}+y^{q}+z^{r}+a x y z,
$$

with a parameter $a(a \neq 0)$ and $p, q, r \in \mathbb{N}$ satisfying the condition $1 / p+1 / q+1 / r$ $<1$. It is known that the Milnor number $\mu$ of the hyperbolic singularity is $p+q+$ $r-1$ (cf. [2, 3]). Note that if $1 / p+1 / q+1 / r=1$, the singularity defined by $f$, called the parabolic singularity, is quasihomogeneous. In this section, we study hyperbolic singularities.

To prove our results, we give $\omega, \mathcal{A} n n_{\mathcal{D}_{X, O}}^{(1)}(\omega), \mathcal{A} n n_{\mathcal{D}_{X, O}}^{(2)}(\omega)$ in an explicit manner. We shall deal with the case where one of the exponents $p, q, r$ is 2 separately from the generic case that all exponents $p, q, r$ are greater than or equal to 3 .

\section{$\S 3.1$. The cases $p, q, r \geq 3$}

Assume $p, q, r \geq 3$. The next result provides a basis for the dual space of the Milnor algebra for a hyperbolic singularity. 
Lemma 3.1. For the function $f=x^{p}+y^{q}+z^{r}+$ axyz with a non-zero parameter $a$ and $p, q, r \geq 3,1 / p+1 / q+1 / r<1$, the vector space $\mathcal{W}_{f}$ is spanned by $p+q+r-1$ algebraic local cohomology classes $\zeta=\psi d x \wedge d y \wedge d z$, where $\psi$ represents the following algebraic local cohomology classes in $\mathcal{H}_{[O]}^{3}\left(\mathcal{O}_{X}\right)$ :

$$
\begin{gathered}
{\left[\frac{1}{x y z}\right], \quad\left[\frac{1}{x^{j} y z}\right] \quad(2 \leq j \leq p-1),} \\
{\left[\frac{1}{x y^{k} z} \quad(2 \leq k \leq q-1), \quad\left[\frac{1}{x y z^{\ell}}\right] \quad(2 \leq \ell \leq r-1),\right.} \\
{\left[\frac{1}{x^{p} y z}-\frac{p}{a} \frac{1}{x y^{2} z^{2}}\right], \quad\left[\frac{1}{x y^{q} z}-\frac{q}{a} \frac{1}{x^{2} y z^{2}}\right], \quad\left[\frac{1}{x y z^{r}}-\frac{r}{a} \frac{1}{x^{2} y^{2} z}\right],} \\
{\left[\frac{1}{x y z^{r+1}}+\frac{r}{q} \frac{1}{x y^{q+1} z}+\frac{r}{p} \frac{1}{x^{p+1} y z}-\frac{r}{a} \frac{1}{x^{2} y^{2} z^{2}}\right] .}
\end{gathered}
$$

Proof. Since the $p+q+r-1$ cohomology classes $\psi \in \mathcal{H}_{[O]}^{3}\left(\mathcal{O}_{X}\right)$ in the statement are linearly independent, it suffices to prove that they are annihilated by the partial derivatives $f_{x}=\partial f / \partial x, f_{y}=\partial f / \partial y$ and $f_{z}=\partial f / \partial z$. It is obvious that the cohomology classes $\left[\frac{1}{x y z}\right],\left[\frac{1}{x^{j} y z}\right](2 \leq j \leq p-1),\left[\frac{1}{x y^{k} z}\right](2 \leq k \leq q-1),\left[\frac{1}{x y z^{\ell}}\right]$ $(2 \leq \ell \leq r-1)$ are annihilated by $f_{x}, f_{y}$ and $f_{z}$. Since $p x^{p-1}\left[\frac{1}{x^{p} y z}-\frac{p}{a} \frac{1}{x y^{2} z^{2}}\right]=$ $p\left[\frac{1}{x y z}\right]$ and $a y z\left[\frac{1}{x^{p} y z}-\frac{p}{a} \frac{1}{x y^{2} z^{2}}\right]=-p\left[\frac{1}{x y z}\right]$, we have $f_{x}\left[\frac{1}{x^{p} y z}-\frac{p}{a} \frac{1}{x y^{2} z^{2}}\right]=0$. It is easy to see that the class $\left[\frac{1}{x^{p} y z}-\frac{p}{a} \frac{1}{x y^{2} z^{2}}\right]$ is also annihilated by $f_{y}$ and $f_{z}$. One can verify in the same manner that the classes $\left[\frac{1}{x y^{q} z}-\frac{q}{a} \frac{1}{x^{2} y z^{2}}\right]$ and $\left[\frac{1}{x y z^{r}}-\frac{r}{a} \frac{1}{x^{2} y^{2} z}\right]$ are annihilated by $f_{x}, f_{y}$ and $f_{z}$. Since $p x^{p-1}\left[\frac{1}{x y z^{r+1}}+\frac{r}{q} \frac{1}{x y^{q+1} z}+\frac{r}{p} \frac{1}{x^{p+1} y z}-\frac{r}{a} \frac{1}{x^{2} y^{2} z^{2}}\right]=$ $\left[r \frac{1}{x^{2} y z}\right]$ and $a y z\left[\frac{1}{x y z^{r+1}}+\frac{r}{q} \frac{1}{x y^{q+1} z}+\frac{r}{p} \frac{1}{x^{p+1} y z}-\frac{r}{a} \frac{1}{x^{2} y^{2} z^{2}}\right]=-r\left[\frac{1}{x^{2} y z}\right], f_{x}$ annihilates the class $\left[\frac{1}{x y z^{r+1}}+\frac{r}{q} \frac{1}{x y^{q+1} z}+\frac{r}{p} \frac{1}{x^{p+1} y z}-\frac{r}{a} \frac{1}{x^{2} y^{2} z^{2}}\right]$. One can also see that $f_{y}$ and $f_{z}$ annihilate that class. Thus, all $p+q+r-1$ classes of the form $\psi d x \wedge d y \wedge d z$ given above belong to $\mathcal{W}_{f}$ and constitute a basis of the vector space $\mathcal{W}_{f}$.

Corollary 3.1. The algebraic local cohomology class

$$
\left[\frac{1}{x y z^{r+1}}+\frac{r}{q} \frac{1}{x y^{q+1} z}+\frac{r}{p} \frac{1}{x^{p+1} y z}-\frac{r}{a} \frac{1}{x^{2} y^{2} z^{2}}\right] d x \wedge d y \wedge d z
$$

in Lemma 3.1 is a generator of $\mathcal{W}_{f}$ over $\mathcal{O}_{X, O}$.

Proof. Denote by $\omega$ the cohomology class (3.1). For each class $\omega^{\prime}$ in Lemma 3.1, one can find a function $h(x, y, z) \in \mathcal{O}_{X, O}$ such that $\omega^{\prime}=h(x, y, z) \omega$.

The notions of algebraic local cohomology classes and the use of Grothendieck local residues provide an answer to the membership problem for the Jacobi ideal $\mathcal{I} \subset \mathcal{O}_{X, O}$ (see [9]). As an application, we have the next result. 
Lemma 3.2. The monomials

$$
1, x^{j}(1 \leq j \leq p-2), \quad y^{k}(1 \leq k \leq q-1), \quad z^{\ell}(1 \leq \ell \leq r), \quad \text { and } \quad z y
$$
constitute a basis of the quotient space $\mathcal{O}_{X, O} / \mathcal{I}$.

Proof. Let $g$ and $g^{\prime}$ be two holomorphic functions defined in a neighborhood of $O$. Then the Grothendieck local duality implies that the difference $g-g^{\prime}$ is in the Jacobi ideal $\mathcal{I}$ if and only if

$$
\operatorname{res}_{O}(g \psi d x \wedge d y \wedge d z)=\operatorname{res}_{O}\left(g^{\prime} \psi d x \wedge d y \wedge d z\right)
$$

for every algebraic local cohomology classes $\psi$ given in Lemma 3.1, where $\operatorname{res}_{O}(*)$ denotes the Grothendieck local residue at $O$. It follows that any element in the Milnor algebra $\mathcal{O}_{X, O} / \mathcal{I}$ can be uniquely represented as a linear combination of the monomials (3.2). This completes the proof.

The next result gives a characterization of annihilators in $L_{\mathcal{D}_{X}}^{(1)}(\omega)$.

Proposition 3.1 ([5, 8, 9]). Let $A$ be a linear partial differential operator of order 1 with coefficients in $B$. The following conditions are equivalent:

(1) There exists $h \in \mathcal{O}_{X, O}$ such that $A+h \in L_{\mathcal{D}_{X}}^{(1)}(\omega)$.

(2) $A g \in \mathcal{I}$ for any $g \in \mathcal{I}$.

Proposition 3.1 provides an effective method [9] for constructing first order annihilators in $L_{\mathcal{D}_{X}}^{(1)}(\omega)$. We fix a term ordering $\frac{\partial}{\partial x} \succ \frac{\partial}{\partial y} \succ \frac{\partial}{\partial z}$.

Lemma 3.3. Let $f=x^{p}+y^{q}+z^{r}+a x y z, a \neq 0$, and let $\omega$ be the generator (3.1). Then $L_{\mathcal{D}_{X}}^{(1)}(\omega)$ is spanned by the following $p+q+r$ first-order differential operators:

- $x^{2} \frac{\partial}{\partial x}-\frac{(p-1) q}{a} y^{q-1} \frac{\partial}{\partial z}-(p-1) x \quad(p \geq 4)$,

- $x^{j} \frac{\partial}{\partial x}-(p+1-j) x^{j-1}, \quad j=3, \ldots, p-2(p \geq 4)$,

- $y^{q-1} \frac{\partial}{\partial x}-\frac{a}{q(r-1)} z^{2} \frac{\partial}{\partial z}+\frac{a}{q} z$

- $p \geq 4: y z \frac{\partial}{\partial x}+2 \frac{p}{a} x^{p-2}$, $p=3: y z \frac{\partial}{\partial x}+\frac{6 q}{a^{2}} y^{q-1} \frac{\partial}{\partial z}+\frac{6}{a} x$

- $z^{r-1} \frac{\partial}{\partial x}+\frac{a}{r} y z \frac{\partial}{\partial z}$

- $z^{r} \frac{\partial}{\partial x}+\frac{a}{r} y z$ 
- $y^{2} \frac{\partial}{\partial y}+(q-1) y z \frac{\partial}{\partial z}-(q-1) y$

- $y^{k} \frac{\partial}{\partial y}-(q+1-k) y^{k-1}, \quad k=3, \ldots, q-1(q \geq 4)$

- $y z \frac{\partial}{\partial y}+\frac{1}{r-1} z^{2} \frac{\partial}{\partial z}-z$

- $z^{r-1} \frac{\partial}{\partial y}-\frac{q}{r} y^{q-1} \frac{\partial}{\partial z}$

- $z^{r} \frac{\partial}{\partial y}-\frac{q}{r} y^{q-1}$

- $z^{\ell} \frac{\partial}{\partial z}-(r+1-\ell) z^{\ell-1}, \quad \ell=3, \ldots, r$.

Proof. It is easy to see by direct computations that these operators annihilate $\omega$. Let $M$ be the set of monomials (3.2). Let $M_{x}, M_{y}, M_{z}$ be the sets of residual monomials in $M \backslash\{1\}$ of the coefficients of the leading terms of the above operators:

$$
\begin{aligned}
& M_{x}=\left\{x, y^{k}(1 \leq k \leq q-1), z^{\ell}(1 \leq \ell \leq r)\right\}, \\
& M_{y}=\left\{y, x^{j}(1 \leq j \leq p-2), z^{\ell}(1 \leq \ell \leq r)\right\}, \\
& M_{z}=\left\{z, z^{2}, x^{j}(1 \leq j \leq p-2), y^{k}(1 \leq k \leq q-1), y z\right\} .
\end{aligned}
$$

Set

$$
P=\left(\sum_{\lambda \in E_{x}} \alpha_{\lambda} \mathbf{x}^{\lambda}\right) \frac{\partial}{\partial x}+\left(\sum_{\lambda \in E_{y}} \beta_{\lambda} \mathbf{x}^{\lambda}\right) \frac{\partial}{\partial y}+\left(\sum_{\lambda \in E_{z}} \gamma_{\lambda} \mathbf{x}^{\lambda}\right) \frac{\partial}{\partial z}+\sum_{\lambda \in E} c_{\lambda} \mathbf{x}^{\lambda}
$$

where $E_{x}, E_{y}, E_{z}$ and $E$ are the sets of exponents of monomials in $M_{x}, M_{y}, M_{z}$ and $M$, respectively, $\alpha_{\lambda}, \beta_{\lambda}, \gamma_{\lambda}$ and $c_{\lambda}$ are undetermined coefficients, and $\mathbf{x}^{\lambda}=x^{j} y^{k} z^{\ell}$ for $\lambda=(j, k, \ell)$. Assume that $\omega P=0$. Then

$$
\left\{\begin{array}{l}
p \alpha_{100}+c_{000}=0 \\
q \beta_{010}+c_{000}=0 \\
r \gamma_{001}+c_{000}=0 \\
\alpha_{100}+\beta_{010}+\gamma_{001}+c_{000}=0
\end{array}\right.
$$

and $\alpha_{j k \ell}=0$ for $(j, k, \ell) \neq(1,0,0), \beta_{j k \ell}=0$ for $(j, k, \ell) \neq(0,1,0), \gamma_{j k \ell}=0$ for $(j, k, \ell) \neq(0,0,1)$, and $c_{j k \ell}=0$ for $(j, k, \ell) \neq(0,0,0)$.

Thus $\left(\alpha_{100}, \beta_{010}, \gamma_{001}, c_{000}\right) \neq(0,0,0,0)$ only when $1 / p+1 / q+1 / r=1$, which contradicts the hyperbolicity.

Notice that for instance the second operator in Lemma 3.3 can be constructed from the first one by multiplying with $x^{j-2}$ from the right. Thus, to compute 
$S o l_{f, \omega}^{(1)}$, one does not need to solve the differential equations $\zeta P=0$ for all operators in $L_{\mathcal{D}_{X}}^{(1)}(\omega)$. Actually, by solving the system $\zeta P=0$ for the third, seventh and first $(p \geq 4)$ or fourth $(p=3)$ operators, we obtain for $p, q, r \geq 3$ the following result, which is the first statement of Theorem 2.1 for the hyperbolic case.

Theorem 3.1. Let $f$ be a hyperbolic singularity and $\omega$ be a generator of $\mathcal{W}_{f}$. Then $\operatorname{Sol}_{f, \omega}^{(1)}=\operatorname{Span}_{\mathbb{C}}\{\delta, \omega\}$.

Proof. We give the proof for $p, q, r \geq 4$. The case $p=3$ can be proved in a similar manner.

Let $P_{1}, P_{3}, P_{7}$ be the first, third and seventh operators in Lemma 3.3. We verify that

$$
\operatorname{Span}_{\mathbb{C}}\left\{\zeta \in \mathcal{W}_{f} \mid \zeta P_{1}=\zeta P_{3}=\zeta P_{7}=0\right\}=\operatorname{Span}_{\mathbb{C}}\{\delta, \omega\} .
$$

Since the action of a differential operator $P$ from the right on a differential form $\psi d x \wedge d y \wedge d z$ is given by $\left(P^{*} \psi\right) d x \wedge d y \wedge d z$, we compute $P^{*} \psi$ for each $\psi$ in Lemma 3.1 where $P^{*}$ is the formal adjoint operator of $P$.

For

$$
P_{1}=x^{2} \frac{\partial}{\partial x}-\frac{(p-1) q}{a} y^{q-1} \frac{\partial}{\partial z}-(p-1) x
$$

we have

$$
P_{1}^{*}=\left(-\frac{\partial}{\partial x}\right) x^{2}-\frac{(p-1) q}{a}\left(-\frac{\partial}{\partial z}\right) y^{q-1}-(p-1) x .
$$

$P_{1}^{*} \psi$ for each $\psi$ in Lemma 3.1 is given below:

- $P_{1}^{*}\left[\frac{1}{x y z}\right]=0$,

- $P_{1}^{*}\left[\frac{1}{x^{2} y z}\right]=-(p-1)\left[\frac{1}{x y z}\right]$,

- $P_{1}^{*}\left[\frac{1}{x^{j} y z}\right]=\left(-\frac{\partial}{\partial x}\right)\left[\frac{1}{x^{j-2} y z}\right]-(p-1)\left[\frac{1}{x^{j-2} y z}\right]=(j-p-1)\left[\frac{1}{x^{j-1} y z}\right]$ $(3 \leq j \leq p-2)$,

- $P_{1}^{*}\left[\frac{1}{x y^{k} z}\right]=0 \quad(2 \leq k \leq p-1)$,

- $P_{1}^{*}\left[\frac{1}{x y z^{\ell}}\right]=0 \quad(2 \leq \ell \leq r-1)$,

- $P_{1}^{*}\left(\left[\frac{1}{x^{p} y z}-\frac{p}{a} \frac{1}{x y^{2} z^{2}}\right]\right)=(p-1)\left[\frac{1}{x^{p-1} y z}\right]-(p-1)\left[\frac{1}{x^{p-1} y z}\right]=-\left[\frac{1}{x^{p-1} y z}\right]$,

- $P_{1}^{*}\left(\left[\frac{1}{x y^{q} z}-\frac{q}{a} \frac{1}{x^{2} y z^{2}}\right]\right)=-\frac{(p-1) q}{a}\left[\frac{1}{x y z^{2}}\right]+\frac{(p-1) q}{a}\left[\frac{1}{x y z^{2}}\right]=0$, 
- $P_{1}^{*}\left(\left[\frac{1}{x y z^{r}}-\frac{r}{a} \frac{1}{x^{2} y^{2} z}\right]\right)=\frac{r(p-1)}{a}\left[\frac{1}{x y^{2} z}\right]$,

- $P_{1}^{*}\left(\left[\frac{1}{x y z^{r+1}}+\frac{r}{q} \frac{1}{x y^{q+1} z}+\frac{r}{p} \frac{1}{x^{p+1} y z}-\frac{r}{a} \frac{1}{x^{2} y^{2} z^{2}}\right]\right)$

$$
=\frac{(p-1) r}{p}\left[\frac{1}{x^{p} y z}\right]-\frac{(p-1) r}{a}\left[\frac{1}{x y^{2} z^{2}}\right]-\frac{(p-1) r}{p}\left[\frac{1}{x^{p} y z}\right]+\frac{(p-1) r}{a}\left[\frac{1}{x y^{2} z^{2}}\right]=0 .
$$

It follows from the above computations that the solution space for the operator $P_{1}$ in $\mathcal{W}_{f}$ is spanned by $\omega, \delta$ and $\psi d x \wedge d y \wedge d z$ where $\psi$ represents the following algebraic local cohomology classes:

$$
\left[\frac{1}{x y^{k} z}\right] \quad(2 \leq k \leq q-1), \quad\left[\frac{1}{x y z^{\ell}}\right] \quad(2 \leq \ell \leq r-1), \quad\left[\frac{1}{x y^{q} z}\right]-\frac{q}{a}\left[\frac{1}{x^{2} y z^{2}}\right] .
$$

Computing $P_{3}^{*} \psi$ for each $\psi$ in (3.3) in the same manner, we find that the solution space $\left\{\zeta \in \mathcal{W}_{f} \mid \zeta P_{1}=\zeta P_{3}=0\right\}$ is spanned by $\omega, \delta$ and $\psi d x \wedge d y \wedge d z$ with $\psi$ being the following algebraic local cohomology classes:

$$
\left[\frac{1}{x y^{k} z}\right] \quad(2 \leq k \leq q-1), \quad\left[\frac{1}{x y^{q} z}\right]-\frac{q}{a}\left[\frac{1}{x^{2} y z^{2}}\right] .
$$

Finally, computing $P_{7}^{*} \psi$ for each $\psi$ in (3.4), we find that the solution space of the system of differential equations $\zeta P_{1}=\zeta P_{3}=\zeta P_{7}=0$ is spanned by $\omega$ and $\delta$.

Since $S o l(1) \supseteq \operatorname{Span}_{\mathbb{C}, \omega}\{\delta, \omega\}$, this completes the proof.

Lemma 3.4. Let $p, q, r \geq 3$. Then the second-order partial differential operator

$$
R=z^{2} \frac{\partial^{2}}{\partial z^{2}}-c q x \frac{\partial}{\partial x}-c p y \frac{\partial}{\partial y}-c(p q-p-q) z \frac{\partial}{\partial z}+c p q
$$

with $c=\frac{r(r-1)}{p q r-p q-q r-p r}$ is in $L_{\mathcal{D}_{X}}^{(2)}(\omega)$.

Proof. It is easy to see by direct computations that $R$ annihilates $\omega$.

For $p, q, r \geq 3$, we have the following theorem.

Theorem 3.2. Let $f$ be a hyperbolic singularity and $\omega$ be a generator of $\mathcal{W}_{f}$. Then $\operatorname{Sol}_{f, \omega}^{(2)}=\operatorname{Span}_{\mathbb{C}}\{\omega\}$.

Proof. Since the operator (3.6) gives $\delta R=c p q \delta$, we have $\operatorname{Sol}_{f, \omega}^{(2)}=\operatorname{Span}_{\mathbb{C}}\{\omega\}$.

\section{$\S 3.2$. The case $p=2$}

To finish the proof of Theorems 3.1 and 3.2, we now examine the case where one of the exponents $p, q, r$ is equal to 2 . We may assume without loss of generality that $p=2$ and $3 \leq q \leq r$. We give $\mathcal{W}_{f}, \omega, \mathcal{A} n n_{\mathcal{D}_{X}}^{(1)}(\omega), \mathcal{A} n n_{\mathcal{D}_{X}}^{(2)}(\omega)$ in an explicit manner for seven cases of $q$ and $r$. 
3.2.1. The case $q \geq 5$. When $p=2$ and $q \geq 5, \mathcal{W}_{f}, \mathcal{A} n n_{\mathcal{D}_{X}}^{(1)}(\omega)$ and $\mathcal{A} n n_{\mathcal{D}_{X}}^{(1)}(\omega)$ are given in the following results.

Lemma 3.5. For the function $f=x^{2}+y^{q}+z^{r}+a x y z$ with a non-zero parameter $a$, and $5 \leq q \leq r$, the vector space $\mathcal{W}_{f}$ is spanned by $q+r+1$ algebraic local cohomology classes $\zeta=\psi d x \wedge d y \wedge d z$, where $\psi$ represents the algebraic local cohomology classes in $\mathcal{H}_{[O]}^{3}\left(\mathcal{O}_{X}\right)$ given below:

$$
\begin{gathered}
{\left[\frac{1}{x y z}\right], \quad\left[\frac{1}{x y^{k} z}\right] \quad(2 \leq k \leq q-1), \quad\left[\frac{1}{x y z^{\ell}}\right] \quad(2 \leq \ell \leq r-1)} \\
{\left[\frac{1}{x^{2} y z}-\frac{2}{a} \frac{1}{x y^{2} z^{2}}\right], \quad\left[\frac{1}{x y^{q} z}-\frac{q}{a} \frac{1}{x^{2} y z^{2}}+\frac{2 q}{a^{2}} \frac{1}{x y^{2} z^{3}}\right]} \\
{\left[\frac{1}{x y z^{r}}-\frac{r}{a} \frac{1}{x^{2} y^{2} z}+\frac{2 r^{2}}{a^{2}} \frac{1}{x y^{3} z^{2}}\right]}
\end{gathered}
$$

and

$$
\eta=\left[\frac{1}{x y z^{r+1}}+\frac{r}{2} \frac{1}{x^{3} y z}+\frac{r}{q} \frac{1}{x y^{q+1} z}-\frac{r}{a} \frac{1}{x^{2} y^{2} z^{2}}+\frac{2 r}{a^{2}} \frac{1}{x y^{3} z^{3}}\right] .
$$

The algebraic local cohomology class $\eta d x \wedge d y \wedge d z$ is a generator of $\mathcal{W}_{f}$ over $\mathcal{O}_{X, O}$.

Lemma 3.6. Let $f=x^{2}+y^{q}+z^{r}+$ axyz, $a \neq 0(5 \leq q \leq r)$. For a generator

$$
\omega=\left[\frac{1}{x y z^{r+1}}+\frac{r}{2} \frac{1}{x^{3} y z}+\frac{r}{q} \frac{1}{x y^{q+1} z}-\frac{r}{a} \frac{1}{x^{2} y^{2} z^{2}}+\frac{2 r}{a^{2}} \frac{1}{x y^{3} z^{3}}\right] d x \wedge d y \wedge d z
$$

of $\mathcal{W}_{f}, L_{\mathcal{D}_{X}}^{(1)}(\omega)$ is spanned by the following $2+q+r$ first-order differential operators:

- $\theta_{(q, r), 1}=y z^{2} \frac{\partial}{\partial x}+\left(-\frac{2^{2} q(r-2)}{(r-1) a^{3}} y^{q-2}-\frac{2}{(r-1) a} z^{2}\right) \frac{\partial}{\partial z}+\frac{2}{a} z$

- $\theta_{(*, r), 2}=z^{r-1} \frac{\partial}{\partial x}-\frac{2^{2}}{a} z^{r-2} \frac{\partial}{\partial y}+\frac{1}{r} a y z \frac{\partial}{\partial z}$,

- $\theta_{(*, r), 3}=z^{r} \frac{\partial}{\partial x}-\frac{a}{r} y z^{2} \frac{\partial}{\partial z}+\frac{a}{r} y z$

- $\theta_{(q, r), 4}=\left(y^{2}-\frac{2 q r}{a^{2}} z^{r-2}\right) \frac{\partial}{\partial y}+(q-1) y z \frac{\partial}{\partial z}-(q-1) y$,

- $\theta_{(q, r), 5}=y^{3} \frac{\partial}{\partial y}-(q-2) y^{2}+\frac{2(q-2) r}{a^{2}} z^{r-2}$,

- $\theta_{(q, *), 6}^{(k)}=y^{k} \frac{\partial}{\partial y}-(q+1-k) y^{k-1}, \quad k=4, \ldots, q-2(q \geq 6)$,

- $\theta_{(q, r), 7}=y z \frac{\partial}{\partial y}+\left(-\frac{2 q r}{(r-1) a^{2}} y^{q-2}+\frac{1}{r-1} z^{2}\right) \frac{\partial}{\partial z}-z$, 
- $\theta_{(q, *), 8}=y z^{2} \frac{\partial}{\partial y}-\frac{2^{2} q}{a^{2}} y^{q-2}$

- $\theta_{(*, r), 9}=z^{r-1} \frac{\partial}{\partial y}-\frac{1}{2 r} a^{2} y z^{2} \frac{\partial}{\partial z}$

- $\theta_{(*, r), 10}=z^{r} \frac{\partial}{\partial y}-\frac{1}{2 r} a^{2} y z^{2}$,

- $\theta_{(q, r), 11}=z^{3} \frac{\partial}{\partial z}+\frac{2 q(r-2)}{a^{2}} y^{q-2}-(r-2) z^{2}$,

- $\theta_{(*, r), 12}^{(\ell)}=z^{\ell} \frac{\partial}{\partial z}-(r+1-\ell) z^{\ell-1}, \quad \ell=4, \ldots, r$.

The coefficients of the operators in the above lemma are in

$$
B=\operatorname{Span}_{\mathbb{C}}\left\{1, y, y^{2}, \ldots, y^{q-2}, y z, y z^{2}, z, z^{2}, \ldots, z^{r}\right\}
$$

Note that the solution space $\operatorname{Sol}_{f, \omega}^{(1)}$ can be determined by solving only two equations, $\zeta \theta_{(q, r), 1}=0$ and $\zeta \theta_{(q, r), 4}=0$.

Lemma 3.7. Let $f=x^{2}+y^{q}+z^{r}+a x y z, a \neq 0(q \geq 5, r \geq q)$. The second-order partial differential operator

(3.6) $S=\left(y^{q-2}-\frac{1}{2 q a^{2}} z^{2}\right) \frac{\partial^{2}}{\partial z^{2}}-\frac{c a^{3}}{2^{2}} y z \frac{\partial}{\partial x}+\frac{c a^{2}}{q} y \frac{\partial}{\partial y}+\frac{(q-2) c a^{2}}{2 q} z \frac{\partial}{\partial z}-c a^{2}$

with $c=\frac{r(r-1)}{q r-2(q+r)}$ is in $L_{\mathcal{D}_{X}}^{(2)}(\omega)$.

3.2.2. The case $q=4$. Let $f=x^{2}+y^{4}+z^{r}+a x y z$ with $r \geq 5$. A basis of the vector space $\mathcal{W}_{f}$ is the same as for $q \geq 5$. The second-order partial differential operator (3.6) also belongs to $L_{\mathcal{D}_{X}}^{(2)}(\omega)$ in this case. We now give $L_{\mathcal{D}_{X}}^{(1)}(\omega)$ :

Lemma 3.8. Let $f=x^{2}+y^{4}+z^{r}+a x y z, a \neq 0(r \geq 5)$. For the generator

$$
\omega=\left[\frac{1}{x y z^{r+1}}+\frac{r}{2} \frac{1}{x^{3} y z}+\frac{r}{4} \frac{1}{x y^{5} z}-\frac{r}{a} \frac{1}{x^{2} y^{2} z^{2}}+\frac{2 r}{a^{2}} \frac{1}{x y^{3} z^{3}}\right] d x \wedge d y \wedge d z
$$

of $\mathcal{W}_{f}, L_{\mathcal{D}_{X}}^{(1)}(\omega)$ is spanned by the following $6+r$ first-order differential operators:

- $r \geq 6: \theta_{(4, r), 1}+\frac{2^{5} 3(r-2) r}{(r-1) a^{5}} z^{2}$

$r=5: \theta_{(4,5), 1}-\frac{2^{6} 3^{2} 5}{a^{7}} y^{2}+\frac{2^{7} 3^{2} 5^{2}}{a^{9}} z^{3}+\frac{2^{3} 3^{2} 5}{a^{5}} z^{2}$,

- $\theta_{(*, r), 2}$,

- $\theta_{(*, r), 3}$, 
- $\theta_{(4, r), 4}$,

- $r \geq 6: \theta_{(4, r), 7}+\frac{2^{4} 3 r^{2}}{(r-1) a^{4}} z^{r-3}$ $r=5: \theta_{(4,5), 7}-\frac{2^{5} 3 \cdot 5^{2}}{a^{6}} y^{2}+\frac{2^{6} 3 \cdot 5^{3}}{a^{8}} z^{3}+\frac{2^{2} 3 \cdot 5^{2}}{a^{4}} z^{2}$,

- $\theta_{(4, *), 8}+\frac{2^{5} r}{a^{4}} z^{r-2}$

- $\theta_{(*, r), 9}$,

- $\theta_{(*, r), 10}$,

- $\theta_{(4, r), 11}-\frac{2^{4}(r-2) r}{a^{4}} z^{r-2}$

- $\theta_{(*, r), 12}^{(\ell)}, \ell=4, \ldots, r$.

The coefficients of the operators in the above lemma are in

$$
B=\operatorname{Span}_{\mathbb{C}}\left\{1, y, y^{2}, y z, y z^{2}, z, z^{2}, \ldots, z^{r}\right\}
$$

Note that the solution space $S o l_{f, \omega}^{(1)}$ can be determined by solving only two equations, $\zeta \theta_{(q, r), 1}=0$ and $\zeta \theta_{(q, r), 4}=0$.

3.2.3. The case $q=3$. Let $f=x^{2}+y^{3}+z^{r}+a x y z$ with $r \geq 7$.

Lemma 3.9. For the function $f=x^{2}+y^{3}+z^{r}+a x y z$ with a non-zero parameter $a$, $r \geq 7$, the vector space $\mathcal{W}_{f}$ is spanned by $r+4$ algebraic local cohomology classes $\zeta=\psi d x \wedge d y \wedge d z$, where $\psi$ represents the algebraic local cohomology classes in $\mathcal{H}_{[O]}^{3}\left(\mathcal{O}_{X}\right)$ given below:

$$
\begin{gathered}
{\left[\frac{1}{x y z}\right], \quad\left[\frac{1}{x y^{2} z}\right], \quad\left[\frac{1}{x y z^{\ell}}\right] \quad(2 \leq \ell \leq r-1)} \\
{\left[\frac{1}{x^{2} y z}-\frac{2}{a} \frac{1}{x y^{2} z^{2}}\right], \quad\left[\frac{1}{x y^{3} z}-\frac{3}{a} \frac{1}{x^{2} y z^{2}}+\frac{6}{a^{2}} \frac{1}{x y^{2} z^{3}}\right],} \\
{\left[\frac{1}{x y z^{r}}-\frac{r}{a} \frac{1}{x^{2} y^{2} z}+\frac{2 r^{2}}{a^{2}} \frac{1}{x y^{3} z^{2}}-\frac{6 r}{a^{3}} \frac{1}{x^{2} y z^{3}}+\frac{12 r}{a^{4}} \frac{1}{x y^{2} z^{4}}\right]}
\end{gathered}
$$

and

$\eta=\left[\frac{1}{x y z^{r+1}}+\frac{r}{2} \frac{1}{x^{3} y z}+\frac{r}{3} \frac{1}{x y^{4} z}-\frac{r}{a} \frac{1}{x^{2} y^{2} z^{2}}+\frac{2 r}{a^{2}} \frac{1}{x y^{3} z^{3}}-\frac{6 r}{a^{3}} \frac{1}{x^{2} y z^{4}}+\frac{12 r}{a^{4}} \frac{1}{x y^{2} z^{5}}\right]$

The algebraic local cohomology class $\eta d x \wedge d y \wedge d z$ above is a generator of $\mathcal{W}_{f}$ over $\mathcal{O}_{X, O}$ in each case. 
Lemma 3.10. Let $f=x^{2}+y^{3}+z^{r}+a x y z, a \neq 0$. For the generator

$$
\begin{aligned}
\omega= & {\left[\frac{1}{x y z^{r+1}}+\frac{r}{2} \frac{1}{x^{3} y z}+\frac{r}{3} \frac{1}{x y^{4} z}\right.} \\
& \left.\quad-\frac{r}{a} \frac{1}{x^{2} y^{2} z^{2}}+\frac{2 r}{a^{2}} \frac{1}{x y^{3} z^{3}}-\frac{6 r}{a^{3}} \frac{1}{x^{2} y z^{4}}+\frac{12 r}{a^{4}} \frac{1}{x y^{2} z^{5}}\right] d x \wedge d y \wedge d z
\end{aligned}
$$

of $\mathcal{W}_{f}, L_{\mathcal{D}_{X}}^{(1)}(\omega)$ is spanned by the following $5+r$ first-order differential operators:

- $r \geq 10: \theta_{r, 1}=y z^{2} \frac{\partial}{\partial x}-\frac{2^{2}}{3 a} y z \frac{\partial}{\partial y}+\left(-\frac{2^{2}(r-6)}{(r-1) a^{3}} y-\frac{2 \cdot 5}{3(r-1) a} z^{2}\right) \frac{\partial}{\partial z}$,

$$
+\frac{2^{4} 3 \cdot 5 r(r-6)}{(r-1) a^{7}} z^{r-5}+\frac{2 \cdot 5}{3 a} z
$$

$r=9: \theta_{9,1}-\frac{2^{2} 3^{5} 5}{a^{9}} y z^{2}+\frac{2^{4} 3^{8} 5}{a^{13}} z^{7}$,

$r=8: \theta_{8,1}-\frac{2^{9} 3^{2} 5}{7 a^{9}} y z+\frac{2^{14} 3^{3} 5}{7 a^{13}} z^{5}$,

$r=7: \theta_{7,1}-\frac{2^{10} 3^{5} 5 \cdot 7^{3}}{a^{21}} y z^{2}-\frac{2^{7} 3^{3} 5 \cdot 7^{2}}{a^{15}} y z-\frac{2^{4} 3 \cdot 5 \cdot 7}{a^{9}} y$

$$
+\frac{2^{12} 3^{6} 5 \cdot 7^{4}}{a^{25}} z^{5}+\frac{2^{9} 3^{4} 5 \cdot 7^{3}}{a^{19}} z^{4}+\frac{2^{6} 3^{2} 5 \cdot 7^{2}}{a^{13}} z^{3}
$$

- $r \geq 10: \theta_{r, 2}=z^{r-2} \frac{\partial}{\partial x}+\left(-\frac{2}{a} z^{r-3}-\frac{a^{3}}{2 \cdot 3^{2} r} y z\right) \frac{\partial}{\partial y}$

$$
+\left(\frac{a}{3(r-1)} y-\frac{a^{3}}{2 \cdot 3^{2} r(r-1)} z^{2}\right) \frac{\partial}{\partial z}-\frac{2^{2} \cdot 5 r}{(r-1) a^{3}} z^{r-5}+\frac{a^{3}}{2 \cdot 3^{2} r} z,
$$

$r=9: \theta_{9,2}+\frac{3^{3} 5}{a^{5}} y z^{2}-\frac{2^{2} 3^{6} 5}{a^{9}} z^{7}$,

$r=8: \theta_{8,2}+\frac{2^{6} 3 \cdot 5}{7 a^{5}} y z-\frac{2^{11} 3^{2} 5}{7 a^{9}} z^{5}$

$r=7: \theta_{7,2}+\frac{2^{5} 3^{2} 7}{a^{7}} z^{5} \frac{\partial}{\partial y}-\frac{2^{3} 3}{a^{3}} z^{3} \frac{\partial}{\partial z}-\frac{2^{8} 3^{4} 7^{2} 109}{a^{17}} y z^{2}-\frac{2^{5} 3^{2} 7 \cdot 109}{a^{11}} y z-\frac{2^{2} 109}{a^{5}} y$

$$
+\frac{2^{10} 3^{5} 7^{3} 109}{a^{21}} z^{5}+\frac{2^{7} 3^{3} 7^{2} 109}{a^{15}} z^{4}+\frac{2^{4} 3 \cdot 7 \cdot 109}{a^{9}} z^{3}+\frac{2^{3} \cdot 3 \cdot 5}{a^{3}} z^{2},
$$

- $r \geq 9: \theta_{r, 3}=z^{r-1} \frac{\partial}{\partial x}-\frac{2^{2}}{a} z^{r-2} \frac{\partial}{\partial y}+\frac{1}{2 \cdot 3 r} a^{3} z^{3} \frac{\partial}{\partial z}$

$$
+\frac{r-2}{r} a y-\frac{2^{2} 3(r-2)}{a^{3}} z^{r-4}-\frac{r-2}{2 \cdot 3 r} a^{3} z^{2}
$$

$r=8: \theta_{8,3}-\frac{2^{4} 3^{3}}{a^{5}} y z^{2}+\frac{2^{9} 3^{4}}{a^{9}} z^{6}$,

$r=7: \theta_{7,3}+\frac{2^{6} 3^{4} 5 \cdot 7}{a^{11}} y z^{2}+\frac{2^{3} 3^{2} 5}{a^{5}} y z-\frac{2^{8} 3^{5} 5 \cdot 7^{2}}{a^{15}} z^{5}-\frac{2^{5} 3^{3} 5 \cdot 7}{a^{9}} z^{4}$, 
- $r \geq 8: \theta_{r, 4}=z^{r} \frac{\partial}{\partial x}-\frac{1}{2 \cdot 3 r} a^{3} z^{4} \frac{\partial}{\partial z}-\frac{r-4}{r} a y z+\frac{2^{2} 3(r-4)}{a^{3}} z^{r-3}+\frac{r-3}{2 \cdot 3 r} a^{3} z^{3}$, $r=7: \theta_{7,4}-\frac{2^{3} 3^{3}}{a^{5}} y z^{2}+\frac{2^{5} 3^{4} 7}{a^{9}} z^{5}$,

- $r \geq 9: \theta_{r, 5}=\left(y z^{2}-\frac{2^{2} 3^{2} r}{a^{4}} z^{r-2}\right) \frac{\partial}{\partial y}+2 z^{3} \frac{\partial}{\partial z}$

$$
+\frac{2^{2} 3(r-3)}{a^{2}} y-\frac{2^{4} 3^{2}(r-3) r}{a^{6}} z^{r-4}-2(r-2) z^{2}
$$

$r=8: \theta_{8,5}+\frac{2^{8} 3^{3} 5}{a^{8}} y z^{2}-\frac{2^{13} 3^{4} 5}{a^{12}} z^{6}$,

$r=7: \theta_{7,5}+\frac{2^{10} 3^{5} 7^{2}}{a^{14}} y z^{2}+\frac{2^{7} 3^{3} 7}{a^{8}} y z-\frac{2^{12} 3^{6} 7^{3}}{a^{18}} z^{5}-\frac{2^{9} 3^{4} 7^{2}}{a^{12}} z^{4}$,

- $r \geq 8: \theta_{r, 6}=z^{r-1} \frac{\partial}{\partial y}-\frac{1}{2^{2} 3 r} a^{4} z^{4} \frac{\partial}{\partial z}-\frac{r-3}{2 r} a^{2} y z+\frac{2 \cdot 3(r-3)}{a^{2}} z^{r-3}+\frac{r-3}{2^{2} 3 r} a^{4} z^{3}$, $r=7: \theta_{7,6}-\frac{2^{4} 3^{2}}{a^{4}} y z^{2}+\frac{2^{6} 3^{3} 7}{a^{8}} z^{5}$,

- $\theta_{r, 7}=z^{r} \frac{\partial}{\partial y}-\frac{1}{2 r} a^{2} y z^{2}+\frac{2 \cdot 3}{a^{2}} z^{r-2}$,

- $r \geq 9: \theta_{r, 8}=\left(y z-\frac{1}{2 \cdot 3} a^{2} z^{3}\right) \frac{\partial}{\partial z}-(r-2) y+\frac{2^{2} 3 r(r-6)}{a^{4}} z^{r-4}+\frac{r-2}{2 \cdot 3} a^{2} z^{2}$,

$r=8: \theta_{8,8}-\frac{2^{7} 3^{2}}{a^{6}} y z^{2}+\frac{2^{12} 3^{3}}{a^{10}} z^{6}$,

$r=7: \theta_{7,8}-\frac{2^{6} 3^{4} 7^{2}}{a^{12}} y z^{2}-\frac{2^{3} 3^{2} 7}{a^{6}} y z+\frac{2^{8} 3^{5} 7^{3}}{a^{16}} z^{5}+\frac{2^{5} 3^{3} 7^{2}}{a^{10}} z^{4}$,

- $r \geq 8: \theta_{r, 9}=\left(z^{2} y-\frac{1}{2 \cdot 3} a^{2} z^{4}\right) \frac{\partial}{\partial z}-(r-3) y z+\frac{2^{2} 3 r(r-6)}{a^{4}} z^{r-3}+\frac{r-3}{2 \cdot 3} a^{2} z^{3}$, $r=7: \theta_{7,9}-\frac{2^{3} 3^{2} 7}{a^{6}} y z^{2}+\frac{2^{5} 3^{3} 7^{2}}{a^{10}} z^{5}$,

- $\theta_{r, 10}=z^{5} \frac{\partial}{\partial z}+\frac{2 \cdot 3(r-4)}{a^{2}} y z^{2}-\frac{2^{3} 3^{2}(r-4) r}{a^{6}} z^{r-2}-(r-4) z^{4}$,

- $\theta_{r, 11}^{(\ell)}=z^{\ell} \frac{\partial}{\partial z}-(r+1-\ell) z^{\ell-1}, \quad \ell=6, \ldots, r$.

The coefficients of the operators in the above lemma are in

$$
B=\operatorname{Span}_{\mathbb{C}}\left\{1, y, y z, y z^{2}, z, z^{2}, \ldots, z^{r}\right\} .
$$

Note that in order to check $\operatorname{Sol}_{f, \omega}^{(1)}=\operatorname{Span}_{\mathbb{C}}\{\omega, \delta\}$, it is enough to solve only two equations, $\zeta \theta_{r, 1}=0$ and $\zeta \theta_{r, 5}=0$. 
Lemma 3.11. For $q=3$, the following second-order partial differential operator is in $L_{\mathcal{D}_{X}}^{(2)}(\omega)$ :

- For $f=x^{2}+y^{3}+z^{r}+$ axyz with $r \geq 9$,

$$
\begin{aligned}
& z^{4} \frac{\partial^{2}}{\partial x \partial z}+\frac{2^{3}(r+3)(r-6)}{a^{5}} z^{r-2} \frac{\partial^{2}}{\partial y^{2}}-\frac{2\left(r^{2}-2 r-12\right)}{3 r a} z^{3} \frac{\partial^{2}}{\partial y \partial z} \\
& -\frac{2^{3}(r-3)}{r a^{3}} z^{2} \frac{\partial^{2}}{\partial z^{2}}-(r-3) z^{3} \frac{\partial}{\partial x} \\
& +\left(\frac{2^{4} 3 r(r-6)(r-2)}{a^{7}} z^{r-4}+\frac{2(r-2)\left(r^{2}-2 r-12\right)}{3 r a} z^{2}-\frac{2^{2}\left(r^{3}-6 r^{2}-2 r+24\right)}{r a^{3}} y\right) \frac{\partial}{\partial y} \\
& +\frac{2^{3}(r-3)(r+2)}{r a^{3}} z \frac{\partial}{\partial z}-\frac{2^{3} 3(r-3)}{a^{3}}
\end{aligned}
$$

- For $f=x^{2}+y^{3}+z^{8}+a x y z$,

$$
\begin{aligned}
z^{4} \frac{\partial^{2}}{\partial x \partial z} & +\frac{2^{5} 23}{11 a^{5}} z^{6} \frac{\partial^{2}}{\partial y^{2}}+\left(-\frac{2 \cdot 7}{11 a} z^{3}-\frac{2^{6}}{11 a^{3}} y z\right) \frac{\partial^{2}}{\partial y \partial z}-\frac{2 \cdot 3 \cdot 5}{11 a^{3}} z^{2} \frac{\partial^{2}}{\partial z^{2}} \\
& +\left(-5 z^{3}+\frac{2 \cdot 3 \cdot 5^{3}}{11 a^{2}} y z\right) \frac{\partial}{\partial x}+\left(-\frac{2^{2} 3 \cdot 7}{11 a} z^{2}-\frac{2^{3} 7 \cdot 19}{11 a^{3}} y\right) \partial_{y}+\frac{2^{4} 3 \cdot 5 \cdot 7}{11 a^{3}}
\end{aligned}
$$

- For $f=x^{2}+y^{3}+z^{7}+a x y z$,

$$
\begin{aligned}
(y z & \left.+\frac{1}{2 \cdot 11} a^{2} z^{3}\right) \frac{\partial^{2}}{\partial x \partial z}+\left(-\frac{2^{7} 3^{2} 5^{2} 7^{3} 23}{11 a^{15}} z^{6}+\frac{2^{6} 3^{3} 7^{2}}{a^{9}} z^{5}-\frac{2^{2} 3 \cdot 7^{2}}{11 a^{3}} z^{4}-\frac{7}{3^{2} 11} a y z\right) \frac{\partial^{2}}{\partial y^{2}} \\
& +\left(\frac{2^{5} 3 \cdot 5^{2} 7^{2} 23}{11 a^{11}} z^{4}-\frac{2^{3} 7^{2}}{3 a^{5}} z^{3}-\frac{2^{5} 5 \cdot 7^{3}}{11 a^{7}} y z+\frac{7}{3^{3} 11} a z^{2}+\frac{2 \cdot 7}{3^{2} a} y\right) \frac{\partial^{2}}{\partial y \partial z}-\frac{2^{4} 3^{3} 7}{11 a^{7}} z^{2} \frac{\partial^{2}}{\partial z^{2}} \\
& +\left(-\frac{2^{2} 3^{3} 5 \cdot 7}{11 a^{4}} z^{3}+\frac{2^{5} 3^{5} 7}{11 a^{6}} y z-\frac{5}{2 \cdot 11} a^{2} z^{2}+\frac{1}{11} y\right) \frac{\partial}{\partial x}-\frac{2 \cdot 7}{3^{2} \cdot 11} a z \frac{\partial}{\partial y}+\frac{2^{5} 3^{4} 7^{2}}{11 a^{7}} .
\end{aligned}
$$

Theorems 3.1 and 3.2 can be proved for $p=2$ by using the results presented in this subsection. This completes the proof of the main theorem for the hyperbolic case.

\section{§4. Exceptional families}

There are 14 exceptional families of unimodal singularities, $E_{12}, E_{13}, E_{14}, Z_{11}$, $Z_{12}, Z_{13}, W_{12}, W_{13}, Q_{10}, Q_{11}, Q_{12}, S_{11}, S_{12}$, and $U_{12}$. Theorem 2.2 is proved for these exceptional families by performing case-by-case computations. The proof involves the following five steps.

Step 1. Compute the basis of the vector space $\mathcal{W}_{f}$ and find a generator $\omega$ of $\mathcal{W}_{f}$.

Step 2. Compute a monomial basis of $\mathcal{O}_{X, O} / \mathcal{I}$. 
Step 3. Compute generators of $\mathcal{A} n n_{\mathcal{D}_{X, O}}^{(1)}(\omega)$ by constructing a basis of $L_{\mathcal{D}_{X}}^{(1)}(\omega)$.

Step 4. Compute $\operatorname{Sol}_{f, \omega}^{(1)}$ by solving the system of partial differential equations

$$
\zeta P=0, \quad \forall P \in L_{\mathcal{D}_{X}}^{(1)}(\omega), \text { for } \zeta \in \mathcal{W}_{f} .
$$

Step 5. Compute generators of $\mathcal{A} n n_{\mathcal{D}_{X, O}}^{(2)}(\omega)$.

Since the defining function of the normal form of an exceptional family of the unimodal singularity in question contains a parameter, we have extended the previously developed algorithm [10] to handle such parametric cases. Step 1 is executed by the resulting algorithm implemented in the computer algebra system Risa/Asir. Steps 3 and 5 are performed by another algorithm described in [9].

By executing Steps 1 to 4 for each exceptional family of unimodal singularities, we obtain the following.

Theorem 4.1 (cf. [5]). Let $f$ be an exceptional family of unimodal singularities. Then $\operatorname{Sol}_{f, \omega}^{(1)}=\operatorname{Span}_{\mathbb{C}}\{\delta, \omega\}$.

By executing Step 5 for each case, we have the following result.

Lemma 4.1. Let $f$ define an exceptional family of unimodal hypersurface isolated singularities at the origin. Let $\omega$ be a generator of $\mathcal{W}_{f}$. Then there exists in $L_{\mathcal{D}_{X}}^{(2)}(\omega)$ a second-order partial differential operator $S$ such that

$$
S^{(0)} \notin \mathfrak{m},
$$

where $S^{(0)}$ is the zeroth-order part of the differential operator $S$, and $\mathfrak{m}$ is the maximal ideal in $\mathcal{O}_{X, O}$.

Since $\delta S \neq 0$ provided that $S^{(0)} \notin \mathfrak{m}$ for the delta function $\delta$, we have the following.

Theorem 4.2. Let $f$ be an exceptional family of non-quasihomogeneous unimodal isolated singularities and $\omega$ be a generator of $\mathcal{W}_{f}$. Then $S_{0 l}^{(2)}$ is the one-dimensional vector space spanned by $\omega$.

Instead of providing proofs by presenting the full set of data for each exceptional family of unimodal singularities, we restrict ourselves to confirming the results for the $Z_{13}$ singularity.

Example 1 ( $Z_{13}$ singularity). Set $f=x^{3} y+y^{6}+a x y^{5}$ with a non-zero parameter $a$. Then $f$ defines the $Z_{13}$ singularity at the origin. $\mathcal{W}_{f}$ is spanned by the 
cohomology classes $\zeta=\psi d x \wedge d y$, where $\psi$ represents the algebraic local cohomology classes in $\mathcal{H}_{[O]}^{2}\left(\mathcal{O}_{X}\right)$ given below:

$$
\begin{gathered}
{\left[\frac{1}{x y}\right], \quad\left[\frac{1}{x y^{2}}\right], \quad\left[\frac{1}{x^{2} y}\right], \quad\left[\frac{1}{x y^{3}}\right], \quad\left[\frac{1}{x^{2} y^{2}}\right], \quad\left[\frac{1}{x^{3} y}\right], \quad\left[\frac{1}{x y^{4}}\right], \quad\left[\frac{1}{x^{2} y^{3}}\right],} \\
{\left[\frac{1}{x y^{5}}\right], \quad\left[\frac{1}{x^{2} y^{4}}\right], \quad\left[\frac{1}{x y^{6}}-\frac{1}{3} a \frac{1}{x^{3} y^{2}}-6 \frac{1}{x^{4} y}\right], \quad\left[\frac{1}{x^{2} y^{5}}-5 a \frac{1}{x^{4} y}\right]} \\
{\left[\frac{1}{x^{2} y^{6}}-6 \frac{1}{x^{5} y}-\frac{7}{9} a \frac{1}{x y^{7}}-\frac{1}{3} a \frac{1}{x^{4} y^{2}}+\frac{7}{27} a^{2} \frac{1}{x^{3} y^{3}}\right] .}
\end{gathered}
$$

The algebraic local cohomology class

$$
\omega=\left[\frac{1}{x^{2} y^{6}}-6 \frac{1}{x^{5} y}-\frac{7}{9} a \frac{1}{x y^{7}}-\frac{1}{3} a \frac{1}{x^{4} y^{2}}+\frac{7}{27} a^{2} \frac{1}{x^{3} y^{3}}\right] d x \wedge d y
$$

is a generator of $\mathcal{W}_{f}$ over $\mathcal{O}_{X, O}$.

The monomials

$$
1, y, x, y^{2}, x y, x^{2}, y^{3}, x y^{2}, y^{4}, x y^{3}, y^{5}, x y^{4}, y^{6}
$$

constitute a basis of the quotient space $\mathcal{O}_{X, O} / \mathcal{I}$.

Let $B$ be the vector space spanned by monomials (4.1). Taking coefficients from $B$, the algorithm described in [9] outputs the following 16 operators as a basis of the space $L_{\mathcal{D}_{X}}^{(1)}(\omega)$ :

- $x y \frac{\partial}{\partial x}+\left(-\frac{7^{2}}{2^{2} \cdot 3^{3} \cdot 5} a x y+\frac{23}{2^{2} \cdot 3 \cdot 5} y^{2}\right) \frac{\partial}{\partial y}$

$-\frac{2^{2} \cdot 7^{5}}{3^{13} \cdot 5} a^{7} x y^{4}-\frac{2^{2} \cdot 7^{4}}{3^{11} \cdot 5} a^{6} y^{5}+\frac{2^{2} \cdot 7^{3}}{3^{8} \cdot 5} a^{4} x y^{2}+\frac{2^{2} \cdot 7^{2}}{3^{6} \cdot 5} a^{3} y^{3}-\frac{7}{2 \cdot 3^{3} \cdot 5} a x-\frac{2 \cdot 19}{3 \cdot 5} y$,

- $a x^{2} \frac{\partial}{\partial x}+\left(\frac{37}{2^{2} \cdot 3 \cdot 5} a x y+\frac{3}{2^{2} \cdot 5} y^{2}\right) \frac{\partial}{\partial y}$

$-\frac{7^{4} \cdot 11}{3^{11} \cdot 5} a^{7} x y^{4}-\frac{7^{3} \cdot 11}{3^{9} \cdot 5} a^{6} y^{5}+\frac{7^{2} \cdot 11}{3^{6} \cdot 5} a^{4} x y^{2}+\frac{7 \cdot 11}{3^{4} \cdot 5} a^{3} y^{3}-\frac{89}{2 \cdot 3 \cdot 5} a x-\frac{3}{5} y$,

- $x y^{2} \frac{\partial}{\partial x}+\frac{7^{3}}{3^{7}} a^{4} x y^{3}+\frac{2^{3} \cdot 7}{3^{5}} a^{3} y^{4}+\frac{7}{3^{4}} a^{2} x^{2}-\frac{7}{3^{2}} a x y-y^{2}$,

- $a^{2} y^{4} \frac{\partial}{\partial x}+\left(\frac{1}{2^{2}} a x y+\frac{3^{2}}{2^{2}} y^{2}\right) \frac{\partial}{\partial y}$

$-\frac{2 \cdot 7^{4}}{3^{10}} a^{7} x y^{4}-\frac{2 \cdot 7^{3}}{3^{8}} a^{6} y^{5}+\frac{2 \cdot 7^{2}}{3^{5}} a^{4} x y^{2}+\frac{2 \cdot 7}{3^{3}} a^{3} y^{3}+\frac{1}{2} a x-3^{2} y$,

- $x y^{3} \frac{\partial}{\partial x}+\frac{7^{3}}{3^{7}} a^{4} x y^{4}+\frac{7^{2}}{3^{5}} a^{3} y^{5}-\frac{7}{3^{2}} a x y^{2}-y^{3}$,

- $y^{5} \frac{\partial}{\partial x}+\frac{1}{3} x^{2}+\frac{7^{2}}{3^{4}} a^{2} x y^{3}+\frac{2^{3}}{3^{2}} a y^{4}$ 
- $x y^{4} \frac{\partial}{\partial x}-\frac{7}{3^{2}} a x y^{3}-y^{4}$,

- $y^{6} \frac{\partial}{\partial x}+\frac{7^{2}}{3^{4}} a^{2} x y^{4}+\frac{7}{3^{2}} a y^{5}$,

- $\left(a^{2} x^{2}-\frac{3}{2^{2}} a x y-\frac{3^{3}}{2^{2}} y^{2}\right) \frac{\partial}{\partial y}$

$-\frac{2^{2} \cdot 7^{3}}{3^{9}} a^{7} x y^{4}-\frac{2^{2} \cdot 7^{2}}{3^{7}} a^{6} y^{5}+\frac{2^{2} \cdot 7}{3^{4}} a^{4} x y^{2}+\frac{13}{3^{2}} a^{3} y^{3}-\frac{3}{2} a x+3^{3} y$,

- $y^{3} \frac{\partial}{\partial y}-\frac{7^{3}}{3^{7}} a^{4} x y^{3}-\frac{7}{3^{3}} a^{3} y^{4}-\frac{2 \cdot 7}{3^{4}} a^{2} x^{2}+\frac{7}{3^{2}} a x y-3 y^{2}$,

- $x y^{2} \frac{\partial}{\partial y}+\frac{7^{2}}{3^{4}} a^{3} x y^{3}+\frac{23}{3^{3}} a^{2} y^{4}+\frac{2}{3^{2}} a x^{2}-4 x y$,

- $y^{4} \frac{\partial}{\partial y}-\frac{7^{3}}{3^{7}} a^{4} x y^{4}+\frac{7}{3^{2}} a x y^{2} x-\frac{7^{2}}{3^{5}} a^{3} y^{5}-2 y^{3}$,

- $x y^{3} \frac{\partial}{\partial y}+\frac{7^{2}}{3^{4}} a^{3} x y^{4}-3 x y^{2}+\frac{7}{3^{2}} a^{2} y^{5}$,

- $y^{5} \frac{\partial}{\partial y}+\frac{7}{3^{2}} a x y^{3}-y^{4}$,

- $x y^{4} \frac{\partial}{\partial y}-2 x y^{3}$,

- $y^{6} \frac{\partial}{\partial y}+\frac{7}{3^{2}} a x y^{4}$.

By solving the system of partial differential equations

$$
\zeta P=0, \quad \forall P \in L_{\mathcal{D}_{X}}^{(1)}(\omega)
$$

for $\zeta \in \mathcal{W}_{f}$, we have

$$
\operatorname{Sol}_{f, \omega}^{(1)}=\operatorname{Span}_{\mathbb{C}}\{\omega, \delta\} .
$$

Actually, it is enough to consider the first and the nineth operators to determine the algebraic local cohomology solution space.

As for the second-order annihilators, the algorithm outputs five operators. Among them, we find the operator

$$
\begin{aligned}
S= & y^{2} \frac{\partial^{2}}{\partial y^{2}}+\left(\frac{3 \cdot 5}{2} x+\frac{7 \cdot 13}{2 \cdot 3^{3}} a^{2} y^{3}\right) \frac{\partial}{\partial x}+\left(\frac{7}{2 \cdot 3^{2}} a x+\frac{1}{2} y\right) \frac{\partial}{\partial y} \\
& -\frac{5 \cdot 7^{5}}{3^{12}} a^{7} x y^{3}-\frac{5 \cdot 7^{4}}{3^{10}} a^{6} y^{4}+\frac{5 \cdot 7^{3}}{3^{7}} a^{4} x y+\frac{5 \cdot 7^{2}}{3^{5}} a^{3} y^{2}-2 \cdot 3 \cdot 5 .
\end{aligned}
$$


Since the zeroth-order part

$$
S^{(0)}=-\frac{5 \cdot 7^{5}}{3^{12}} a^{7} x y^{3}+\frac{5 \cdot 7^{3}}{3^{7}} a^{4} x y-\frac{5 \cdot 7^{4}}{3^{10}} a^{6} y^{4}+\frac{5 \cdot 7^{2}}{3^{5}} a^{3} y^{2}-2 \cdot 3 \cdot 5
$$

of $S$ is not contained in the maximal ideal $\mathfrak{m}$, we have

$$
S o l_{f, \omega}^{(2)}=\operatorname{Span}_{\mathbb{C}}\{\omega\}
$$

\section{References}

[1] V. I. Arnold, S. M. Gusein-Zade and A. N. Varchenko, Singularities of differentiable maps, Volume I, Monogr. Math. 82, Birkhäuser, Boston, 1985. Zbl 0554.58001 MR 0777682

[2] W. Ebeling, Functions of several complex variables and their singularities, Grad. Stud. Math. 83, Amer. Math. Soc., 2007. Zbl 1188.32001 MR 2319634

[3] A. M. Gabrielov, Dynkin diagrams for unimodal singularities, Functional Anal. Appl. 8 (1973), no. 3, 192-196. Zbl 0304.14010 MR 367274

[4] Y. Nakamura and S. Tajima, A method for constructing holonomic systems for algebraic local cohomology classes with support on a zero dimensional variety, in Mathematical Software (Beijing, 2002), World Sci., 2002, 158-168. Zbl 1059.14007 MR 1932608

[5] Unimodal singularities and differential operators, in Singularités FrancoJaponaises, Sémin. Congr. 10, Soc. Math. France, 2005, 191-208. Zbl 1086.32024 MR 2145955

[6] M. Noro and T. Takeshima, Risa/Asir - a computer algebra system, in ISSAC 1992, P. S. Wang (ed.), ACM Press, 1992, 387-396. Zbl 0964.68597

[7] K. Saito, Quasihomogene isolierte Singularitäten von Hyperflächen, Invent. Math. 14 (1971), 123-142. Zbl 0224.32011 MR 0294699

[8] S. Tajima and Y. Nakamura, Algebraic local cohomology classes attached to quasihomogeneous isolated hypersurface singularities, Publ. RIMS Kyoto Univ. 41 (2005), 1-10. Zbl 1105.32020 MR 2115965

[9] , Annihilating ideals for an algebraic local cohomology class, J. Symbolic Comput. 44 (2009), 435-448. Zbl 1171.32020 MR 2499921

[10] S. Tajima, Y. Nakamura and K. Nabeshima, Standard bases and algebraic local cohomology for zero dimensional ideals, in Singularities - Niigata-Toyama 2007, Adv. Stud. Pure Math. 56, Math. Soc. Japan, 2009, 341-361. Zbl 1194.13020 MR 2604090 